\title{
Conductivity measurement of ionic liquids confined in the nanopores of metal-organic frameworks: a case study for [BMIM][TFSI] in HKUST-1
}

\author{
Zejun Zhang ${ }^{1}$. Chun $\mathrm{Li}^{1}$ · Abhinav Chandresh ${ }^{1} \cdot$ Lars Heinke $^{1}$
}

Received: 31 May 2021 / Accepted: 25 August 2021 / Published online: 9 September 2021

(c) The Author(s) 2021

\begin{abstract}
Nanoporous materials like metal-organic frameworks (MOFs) attract considerable attention as porous host for electrolytes like ionic liquids (ILs). The conductivity and mobility of the ions in the pores are among the key properties and their experimental quantification is of paramount importance. Here, three different approaches for the quantification of the ion conductivity of IL@MOF via electrochemical impedance spectroscopy (EIS) are compared: the material in the form of IL-impregnated MOF powders pressed into pellets between two planar electrodes, MOF films grown on substrates with deposited electrodes loaded with IL by impregnation, and the IL-loaded MOF films where excess IL is removed. Contactangle measurements and EIS data show that the excess IL on the outer MOF surface of the film or pellet results in apparent conductivities, larger than the intrinsic conductivity of the IL@MOF. Removing the excess IL enables the experimental quantification of the intrinsic IL@MOF conductivity.
\end{abstract}

Keywords Ionic liquid $\cdot$ Metal-organic framework $\cdot$ Impedance spectroscopy $\cdot$ Thin films $\cdot$ Ionic conduction

\section{Introduction}

Ionic liquids (ILs) are organic salts with low melting temperatures, typically below room temperature $[1,2]$. ILs possess various exclusive properties like a negligible vapor pressure, excellent thermal stability, non-flammability, high ionic conductivity, large electrochemical windows, and to some extent adjustable physical and chemical properties. As a result, ILs find widespread applications as electrolytes [3-6]. For many uses, solid-state properties are preferred and the fluid nature of ILs limits their practicality due to leakage problem and non-portability. Thus, ILs confined in suitable porous hosts have been established to obtain conductive, hybrid, solid-like materials.

Metal-organic frameworks (MOFs) [7, 8] are a novel class of nanoporous, crystalline hybrid materials which are attractive hosts for IL embedment. For example, ILs

Lars Heinke

lars.heinke@kit.edu

1 Institute of Functional Interfaces (IFG), Karlsruhe Institute of Technology (KIT), Hermann-von-Helmholtz-Platz 1, 76344 Eggenstein-Leopoldshafen, Germany embedded in MOFs enable the control of the phase behavior and of the IL properties via host-guest interactions [9-12]. A key feature of MOFs as hosts for IL is the ionic conductivity of the IL@MOF material. Usually, the IL@MOF material is prepared in the form of powders [13-15] and then pressed into pellets, and the conductivity of the pellet sandwiched between two electrodes is determined by electrochemical impedance spectroscopy (EIS). The IL loading is usually performed by impregnation, that is, soaking the porous MOF with IL. There, it was found that a significant amount of (excess) IL is present on the outer surface of the MOF crystals [15-17]. The excess IL on the external MOF surface is described as "nanowetted interface" [15], and the IL-MOF material is described as "gel like hybrids" [16], where "the sample is observed to be in the liquid phase... and the excess IL flows out" [17]. The excess IL, which can be beneficial for the contacts to the electrodes [15], hinders the accurate determination of the ionic conduction of IL@ MOFs by EIS. It should be noted that the excess IL does not evaporate like other solvents, due to the negligible small vapor pressure. Since the mobility of the ion typically tremendously decreases by the embedment in the nanopores, the excess IL is typically much more mobile than the IL in the MOF pores. As a result, the impedance of the sample 
a)

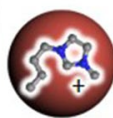
[BMIM]

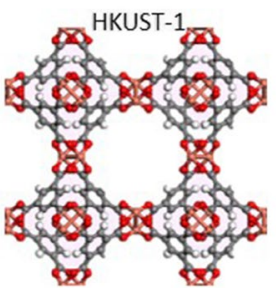

b)

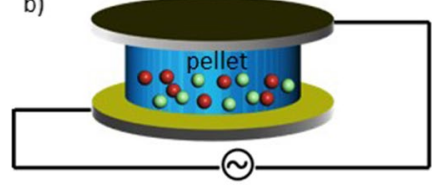

c)

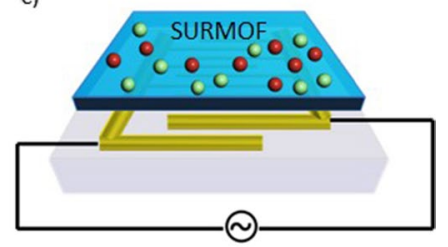

Fig. 1 a Structure of [BMIM][TFSI] ionic liquid and HKUST-1 MOF. $\mathbf{b}$ and $\mathbf{c}$ Sketch of the setup for the conductivity measurement of IL@HKUST-1 MOF material in the form of a pellet sandwiched between two planar electrodes (b) and in the form of a thin film grown on a substrate with deposited electrodes, like interdigitated gold electrodes on glass sheets (c)

measured by EIS is dominated by the highly conducting excess IL in comparison to the IL in the MOF pores, whose comparatively low conductivity barely contributes to the total measured conductivity. An alternative approach is the preparation of substrate-bound MOF films, where the excess IL on the surface upon loading can be washed off by rinsing.

The aim of the present communication is to explore the conduction of IL@MOF and to compare different ways of conductivity measurements. To this end, we use MOF powders impregnated with IL and pressed into pellets for EIS measurements, following standard procedures (approachI). In an alternative approach, thin MOF films prepared in a layer-by-layer fashion on glass substrates with deposited electrodes are used (Fig. 1). The presence of excess IL upon loading (approach-II) is shown by contact angle measurements. In approach-III, the excess IL on the MOF film surface is rinsed off. We use IL of type [BMIM][TFSI] and MOF of type HKUST-1. [BMIM] stands for 1-butyl-3-methylimidazolium, [TFSI] for bis(trifluoromethylsulfonyl)imide, and HKUST-1 stands for Hong Kong University of Science and Technology-1 [18]. Comparing approach-II and -III, we show that the excess IL on the MOF surface substantially increases the determined (apparent) conductivity. Moreover, the determined IL conductivity dependence on the IL@ MOF loading differs for the approaches-I, -II, and -III. In approaches-I and -II, the determined conductivity increases with increasing IL loading, which cannot be explained by a conduction mechanism of IL inside the MOF and which is most likely caused by increasing amounts of excess IL on the MOF surface and between the MOF crystallites, respectively. In approach-III, the determined conductivity decreases with increasing IL loading, in very good agreement with molecular dynamics simulations. Thus, we suggest MOF films prepared on appropriate substrates with deposited electrodes where excess IL is rinsed off as suitable model system for precise quantification of the ionic conductivity of IL inside MOF pores via EIS measurements.

\section{Experimental section}

\section{Synthesis of HKUST-1 powder}

Powder HKUST-1 samples were prepared following a standard synthesis procedure, described in ref. [19]. In detail, $\mathrm{Cu}\left(\mathrm{NO}_{3}\right)_{2} \cdot 3 \mathrm{H}_{2} \mathrm{O}(0.87 \mathrm{~g}, 3.6 \mathrm{mmol})$ and benzene-1,3,5tricarboxylic acid (BTC, $0.42 \mathrm{~g}, 2.0 \mathrm{mmol}$ ) were dissolved in $24 \mathrm{ml}$ of 50:50 vol.\% mixture of ethanol and deionized water. The resulting solution was kept in a Teflon-lined auto-clave at $100{ }^{\circ} \mathrm{C}$ for $24 \mathrm{~h}$. Upon cooling to room temperature, a crystalline powder was found. The powder was centrifuged and washed thoroughly with a mixture of water and ethanol $(50 \% \mathrm{v} / \mathrm{v})$. Finally, the powder was activated under vacuum at $120{ }^{\circ} \mathrm{C}$ for $16 \mathrm{~h}$ and stored in Argon for further experiments.

\section{Loading of ionic liquid in MOF powder and IL@ HKUST-1-pellet preparation}

For the loading of the MOF powder with IL, different amounts of [BMIM][TFSI] IL were added to the activated HKUST-1 powder, homogeneously mixed, and heated under vacuum at $120^{\circ} \mathrm{C}$ overnight. In detail, the IL amounts were $74.9 \mathrm{mg}$ per $100 \mathrm{mg}$ MOF for $100 \%$ loading, $71.1 \mathrm{mg}$ of IL per $100 \mathrm{mg}$ MOF for $95 \%$ loading, $59.9 \mathrm{mg}$ of IL per $100 \mathrm{mg}$ MOF for $80 \%$ loading, $37.4 \mathrm{mg}$ of IL per $100 \mathrm{mg}$ MOF for $50 \%$ loading, and $11.2 \mathrm{mg}$ of IL per $100 \mathrm{mg}$ MOF for $15 \%$ loading. The volume of the amount of IL corresponds to the percentage of the void pore volume in the MOF. For the preparation of the IL@MOF pellets, we followed standard directions [20]. In detail, for each pellet, 200-400 mg of the material was placed inside a stainless steel press and the pellet was formed under a pressure of approximately $150 \mathrm{MPa}$. The resulting pellets had cylindrical shapes of $13 \mathrm{~mm}$ diameter with thicknesses of approximately 1.7-2 mm.

\section{Synthesis of HKUST-1 SURMOF}

The surface-mounted MOF (SURMOF) thin films were prepared in a layer-by-layer (lbl) fashion on glass substrates with deposited interdigitated gold electrodes [21]. In detail, the $\mathrm{lbl}$ growth process consists of alternately exposing the substrate to the ethanolic solutions of the building units, that is, the metal nodes (here, $1 \mathrm{mM}$ copper acetate) and the organic linkers (here: 0.2 mM BTC). Between each immersion step, the sample surfaces were cleaned with 
pure ethanol. The SURMOF samples were prepared in 50 lbl synthesis cycles by using a spray method [21]. Before the SURMOF synthesis, the interdigitated gold electrode substrates were treated by UV-ozone treatment for $15 \mathrm{~min}$ to remove impurities and to increase the number of $\mathrm{OH}$ functional groups and the hydrophilicity. The interdigitated gold electrodes on glass substrates were obtained from DropSens. The total length of the gap between the gold electrodes is $1.69 \mathrm{~m}$ and the gap width is $10 \mu \mathrm{m}$.

\section{Loading of IL in SURMOFs}

The IL loading of the HKUST-1 SURMOFs was performed by immersing the samples in IL/acetonitrile solutions with IL-ratios of $15 \%, 50 \%, 80 \%, 95 \%$, and $100 \%$. The time of the immersion at room temperature was $20 \mathrm{~min}$. Afterwards, a pure nitrogen flow was used to dry the sample and to try to blow off IL from the sample surface (approach-ii). Alternatively, the samples were rinsed with acetonitrile for approximately $2 \mathrm{~s}$ and then dried in a flow of pure nitrogen (approach-iii).

\section{X-ray diffraction and scanning electron microscopy}

The X-ray diffractograms (XRD) were measured in out-ofplane geometry using a Bruker D8-Advance diffractometer equipped with a position-sensitive detector in $\Theta-2 \Theta$ geometry. A Cu-anode with a wavelength of $\lambda=0.154 \mathrm{~nm}$ was used. Scanning electron microscopy (SEM) images were recorded with a TESCAN VEGA3 thermionic emission SEM system. For the cross-section analysis, samples were broken. Prior to SEM analysis, all the samples were coated with a thin platinum layer by sputtering at high vacuum.

\section{Infrared (IR) spectroscopy}

Infrared reflection absorption spectroscopy (IRRAS) was used for recording the IR vibration spectra of the SURMOF samples. A FT-IR spectrometer of type Vertex 80 from Bruker equipped with a grazing incidence reflection unit and a narrow band MCT detector was employed to record the FT-IR data from SURMOF films. The IRRAS chamber was purged with pure nitrogen.

\section{Measurement of ionic conduction properties}

The electrochemical impedance spectra (EIS) were measured with a Zurich Instruments MFIA Impedance Analyzer for a frequency range of $5 \mathrm{MHz}$ to $0.5 \mathrm{~Hz}$. The AC voltage is $300 \mathrm{mV}$. No DC voltage is applied. The SURMOF samples were placed in a home-made Teflon cell where the interdigitated gold electrodes were contacted in a two-probe way.
The electric field between two (interdigitated) electrodes is approximately $0.03 \mathrm{~V} \mathrm{~m}^{-1}$. The cell was purged with pure nitrogen with a flow rate of roughly $250 \mathrm{~mL} \mathrm{~min}{ }^{-1}$. The IL concentration was varied in cycles of $15 \%, 50 \%, 80 \%, 95 \%$, and $100 \%$ IL pore filling and repeated 5 times. The average values with the standard deviation as error bars are shown. The conduction of the pellets was measured by positioning the IL@MOF pellets with different IL loadings between 2 planar electrodes, which are conducting Si wafer with a gold thin film (150 nm thickness). All experiments were performed at room temperature in an atmosphere of pure nitrogen.

\section{Contact Angle}

Contact angle measurements were performed on an attension optical contact angle analysis systems from Biolin Scientific. Droplets of deionized water and diiodomethane, respectively, were placed on the substrate, while recording the images. The image analysis was carried out with the software "one attension" using a Young-Laplace analysis mode. The relative humidity and temperature were $43.3 \%$ and $22.8{ }^{\circ} \mathrm{C}$. Since water and $\mathrm{CH}_{2} \mathrm{I}_{2}$ may destroy the structure of HKUST-1, the measurements were performed at many different pristine positions on the same sample.

\section{Results and discussion}

MOF pellets and MOF thin films were prepared as described in the experimental section. The X-ray diffractograms (XRDs; Fig. 2a) of the samples show that the MOF materials are crystalline with the targeted HKUST-1 structure. The crystallinity of the pristine sample also remains upon IL loading. The relative intensities of the XRD peak change, which is a clear indication for the form factor change, caused by the IL loading in the MOF pores.

The SEM images of the samples are shown in Fig. 2b-e. The SEM images (Fig. $2 b$ and c) show that the MOF powder is made of crystallites of roughly 1 to $3 \mu \mathrm{m}$ extension. The crystal facets of some MOF crystals are visible. The top-view SEM images of the SURMOF films (Fig. 2d), in which the interdigitated gold electrode substrates are visible as bright strips, show the homogenous surface coverage of the substrate with the MOF film. SEM images of the crosssection of a broken sample (Fig. 2e) show a SURMOF film thickness of approximately $250 \mathrm{~nm}$.

For mixing and diluting the IL, acetonitrile (ACN) was chosen as solvent because of its full mixability with [BMIM] [TFSI] and its very small conductivity. Thus, the measured conductivity can be solely attributed to the IL. In a previous study, we found that the percentage of the IL in the IL-ACN solution approximately corresponds to the IL pore filling 
upon impregnation and drying [22]. Acetonitrile is very volatile at room temperature and can evaporate from the MOF pores. The infrared spectra of a SURMOF which was initially loaded with acetonitrile are shown in Fig. 3a. The transient spectra show that acetonitrile desorbs from the MOF film and the release time constant is approximately $25 \mathrm{~min}$. For a sample initially loaded with a solution of IL-ACN 1:1, the transient infrared spectra are shown in Fig. 3b. There, the intensity of the ACN band also decreases quickly, indicating the ACN desorption. The determined time constant is slightly larger, that is, $31 \mathrm{~min}$. The slightly decelerated desorption process is presumably caused by the IL which decreases the ACN diffusion rates. In both cases, i.e. pure
Fig. 2 a X-ray diffractograms of the SURMOF and MOF powder samples. The samples are empty and loaded with IL, as indicated in the plot. For comparison, the calculated XRD of the targeted structure is shown in grey.

The reflexes are indicated. b-e Scanning electron microscopy (SEM) images of $\mathbf{b}$ HKUST-1 powder and c IL@HKUST-1 powder pellet and $\mathbf{d}$ the top view $\left(90^{\circ}\right)$ and $\mathbf{e}$ the cross Sect. $\left(20^{\circ}\right)$ of HKUST-1 SURMOF film. The pictures have different magnifications and the $1-\mu \mathrm{m}$ scale bars are shown a)

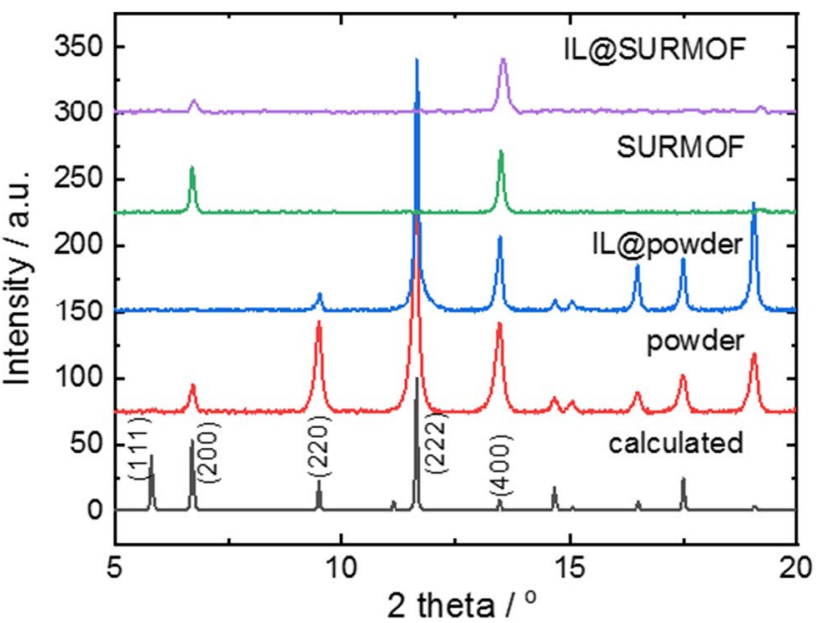

b)
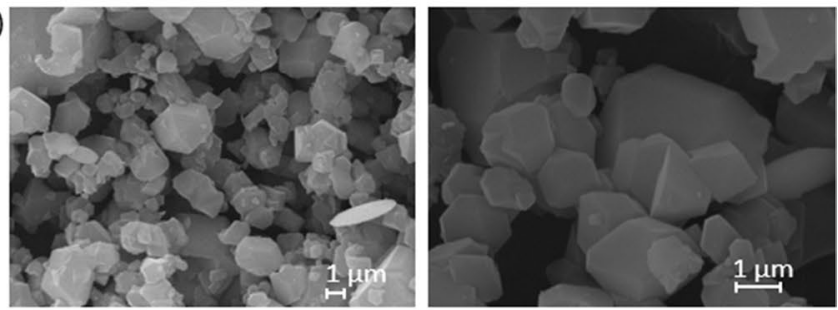

c)
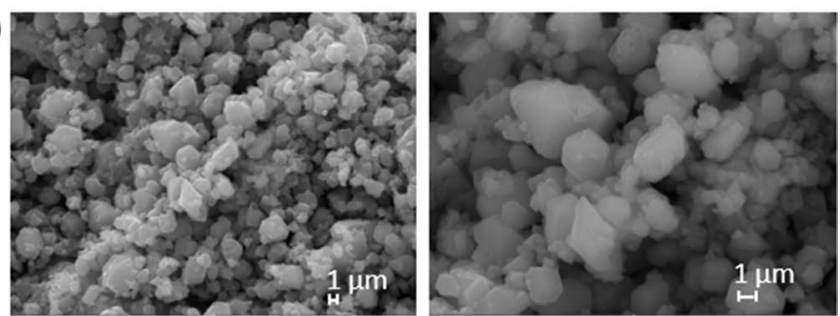

d)
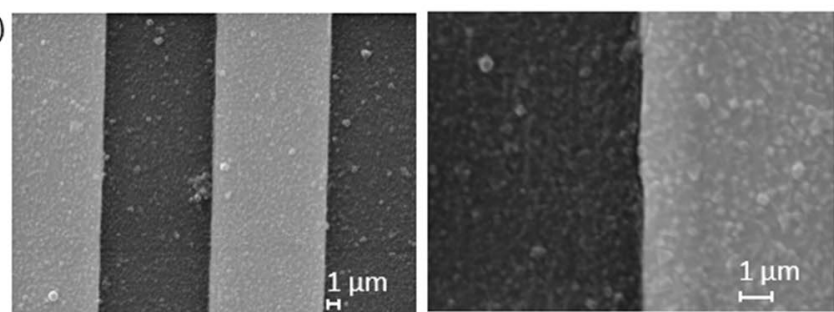

e)

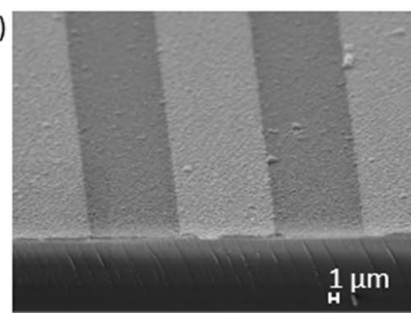


ACN and ACN-IL-mixture, the ACN fully desorbs quickly from the MOF.

After loading the SURMOF with IL via soaking in the ILsolution, additional excess IL can be present on the external sample surface. This excess IL is visible by a slight gloss and cannot be removed by blowing away with pure nitrogen. Contact angle measurements with water and diiodomethane were used to verify the excess IL. For the pristine SURMOF, the contact angle for water and diiodomethane are approximately $52^{\circ}$ and $32^{\circ}$, respectively. Upon loading the SURMOF with IL (without rinsing the surface), the contact angles change to $26^{\circ}$ and $58^{\circ}$, respectively (Fig. 4 and Table S1). The change of the contact angle clearly indicates that the surface of the SURMOF becomes more hydrophilic and less affine towards diiodomethane upon IL loading. Since the contact angle is controlled by the surface termination and not by the pore filling of the MOF, we conclude that there is excess IL on the SURMOF surface. The excess IL on the surface was removed by rinsing with ACN for approximately $2 \mathrm{~s}$. Upon rinsing and drying with nitrogen gas flow, the contact angles are $50^{\circ}$ and $29^{\circ}$ for water and diiodomethane, respectively. These values are very close to the values from the pristine sample. Thus, the contact angle measurements show that there is excess IL on the SURMOF surface upon IL loading and the excess IL can be removed by short $\mathrm{ACN}$ rinsing.

The conductivity of the IL@MOF samples was measured by impedance spectroscopy. Typical Nyquist plots of the samples with different IL-pore fillings are shown in Fig. 5. The data can be described with the appropriate reference circuit, also referred to as $C P E_{\mathrm{geo}} \|\left(R-C P E_{\mathrm{d} 1}\right)$ circuit, shown in the inset. The determined ohmic resistances of the samples with different IL loadings are shown in Figure S1.

Based on the determined ohmic resistances, the sample geometry, and film thicknesses, the conductivity can be calculated, as shown in Fig. 6. The calculated conductivities are in the range of $10^{-7}$ to $10^{-4} \mathrm{~S} \mathrm{~m}^{-1}$, which is many orders of magnitude larger than the conductivity of empty HKUST-1 [23].

For the measurements of the IL@SURMOF without rinsing the surface, this means with excess IL on the outer surface, the calculated conductivity increases when increasing the IL pore filling from 15 to $80 \%$. The same trend, with an even larger magnitude, is also observed for the IL@MOF-pellet samples. The conductivity of the IL@ SURMOF-sample with excess IL as well as for the IL@ MOF-pellet samples decreases when increasing the pore filling from 80 to $100 \%$. For the IL@ SURMOF sample where the excess IL is removed, a different concentration dependence is observed. There, the conductivity decreases monotonically with increasing IL pore filling. This concentration dependence of the conductivity is in very good agreement
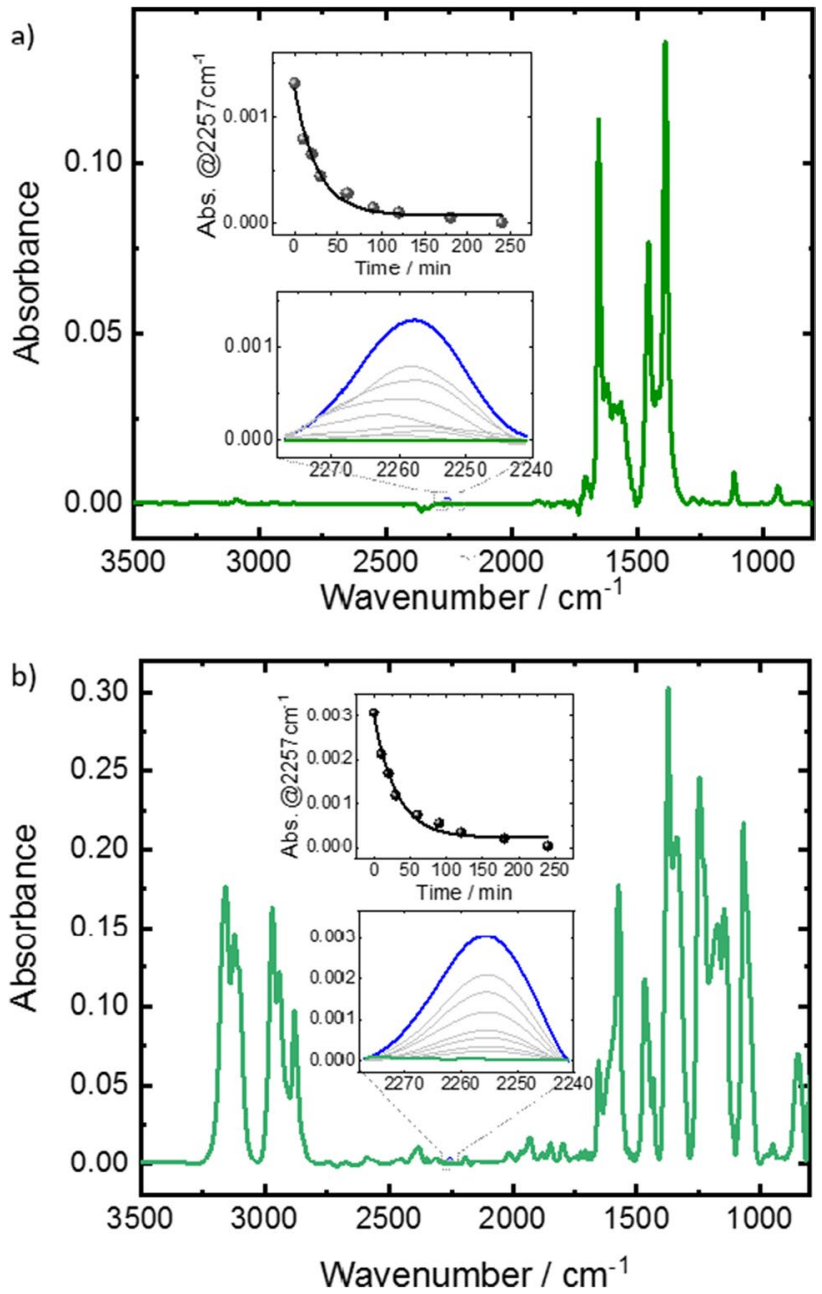

Fig. 3 Infrared reflection absorption spectra of the HKUST-1 SURMOF loaded with pure acetonitrile (a) and with IL-acetonitrile solution (b). The initial spectra of the ACN-loaded sample and the IL$\mathrm{ACN}$-loaded sample are in blue, the final spectra (after $4 \mathrm{~h}$ ) are green, and the spectra in between are plotted in grey. The spectra a virtually identical, except of the band at $2257 \mathrm{~cm}^{-1}$. The insets show the magnification of the band at $2257 \mathrm{~cm}^{-1}$, attributed to $\mathrm{C} \equiv \mathrm{N}$ acetonitrile vibration. The changes of the $\mathrm{C} \equiv \mathrm{N}$ absorbance versus time are also pictured as insets, showing the release of acetonitrile. The time constant of the mono-exponential-decay-functions are $26.5 \mathrm{~min}$ in (a) and $31.4 \mathrm{~min}$ in (b). The bands between 1400 and $1700 \mathrm{~cm}^{-1}$ in (a) are assigned to various vibrations of the MOF linker [9]. For comparison, the IRRA spectra of the sample loaded with $100 \%$ IL, 50\% IL (i.e., IL-ACN 1:1) and of the empty MOF film are shown in Figure S2

with molecular dynamics simulations of the conduction of [BMIM][TFSI] IL in HKUST-1 [22].

By comparing the absolute values of the determined conductivity, at low loadings, the conductivity of the SURMOF sample with and without rinsing the surface is very similar, indicating that there is only a small amount of excess IL on the surface. With nominal increasing IL loading in the pores, the discrepancy becomes larger. We believe that this is caused by the increasing amount of excess IL on the 
Fig. 4 a Sketch of contact angle measurement on the pristine MOF film and on the IL@MOF film before and after rinsing off the excess IL. b Contact angle measurements with deionized water on a pristine HKUST-1 SURMOF film (left), IL-loaded-SURMOF without rinsing (center) and after rinsing (right). The determined contact angles are shown. c Contact angle measurements with diiodomethane for the pristine sample (left), IL-loaded without rinsing (center) and after rinsing (right). The contact angle measurements were repeated several times and the average values and standard deviations are shown in Table S1

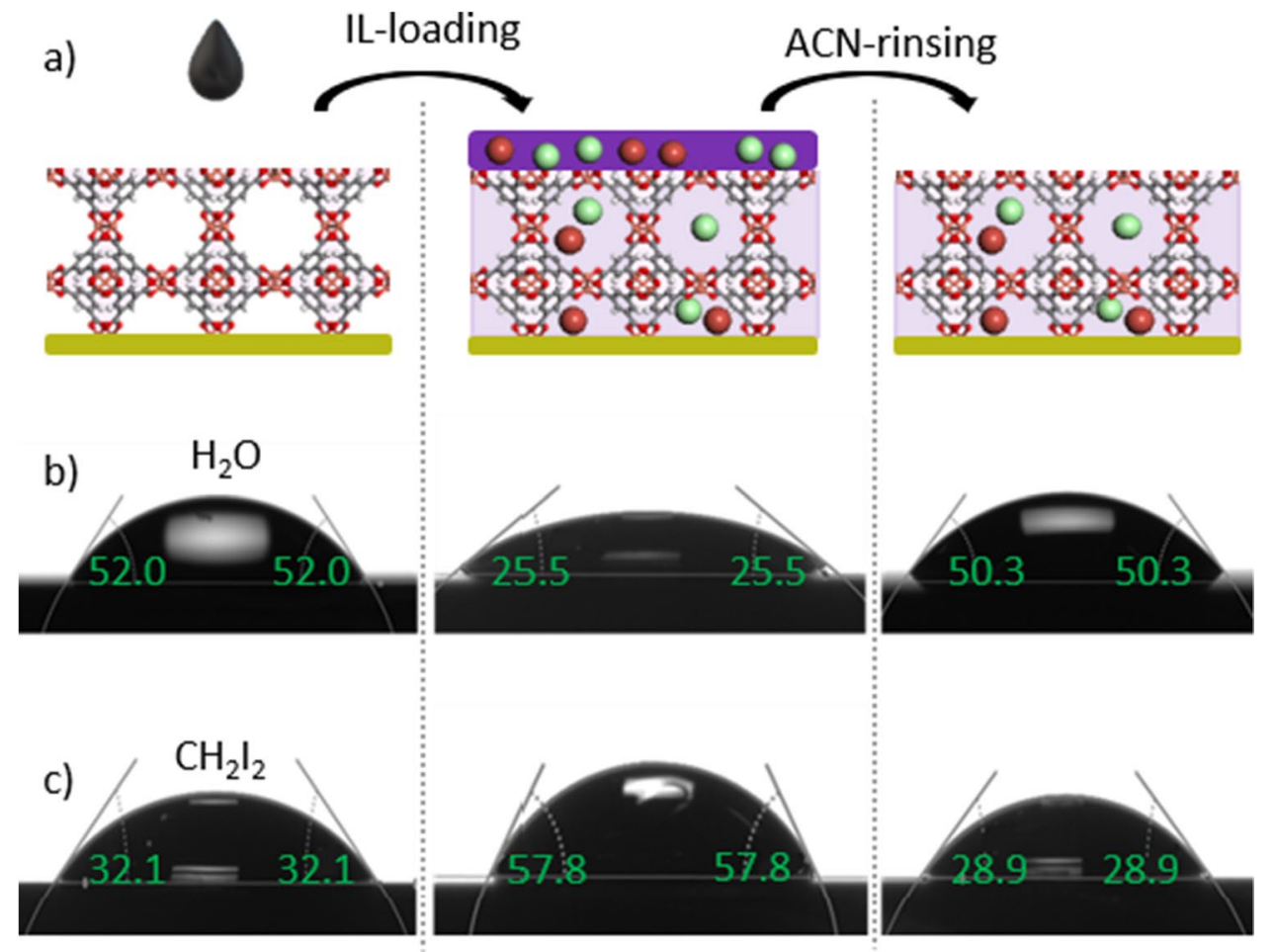

surface. For the IL@MOF-pellet, the increasing conductivity is caused by the increasing amount of excess IL between the MOF crystals in the pellet, also termed "nanowetted interfaces" [15].

Based on this, we conclude that the IL@MOF film where excess IL is removed allows us to determine the intrinsic conductivity of the IL@MOF-material. On the other hand, the IL@MOF films without removing excess IL as well as the pellet samples show apparent conductivities which are larger than the intrinsic conductivity, caused by the additional highly mobile excess IL. It should be noted that the divergence between the conductivities determined with the different approaches is approximately a factor 5 for small loading and a factor of 250 at maximum loading.

\section{Conclusions}

In conclusion, three methods of measuring the conductivity of IL@MOF material are compared. Different concentrations of [BMIM][TFSI] were loaded into HKUST-1 MOF films and MOF pellets via a solution impregnation method or homogeneous mixing and heating. X-ray diffraction form factor changes indicate the successful loading in the MOF pores. Contact angle measurements show that IL is present on the MOF surface upon IL loading. Moreover, the contact angle measurements show that the excess IL can be removed from the outer SURMOF surface by briefly rinsing. For determining the ionic conductivity in MOFs via impedance spectroscopy, the three approaches, that is, pellets of IL-loaded MOFs and ILloaded MOF films without and with surface cleaning upon loading, are compared, showing substantially different results. While the excess IL on the external MOF surface results in apparent large conductivities for the pellets and thin films without surface cleaning, the conductivity of the IL-loaded MOF film with removed excess IL allows us to determine the intrinsic conductivity of the IL under the MOF confinement.

We believe the method can also be applied to most MOF films made in many different ways [24], not just made via layer-by-layer syntheses, provided that the material is bound to a substrate and the surface can be rinsed. Due to the negligible small vapor pressure of ionic liquids, excess IL does not evaporate as common solvents. We foresee that the improvement of the experimental exploration of the conduction of IL in nanoporous MOFs contributes to a better understanding and enhanced performance of various electrolytes in nanoporous confinement, as applied in batteries and supercapacitors. 

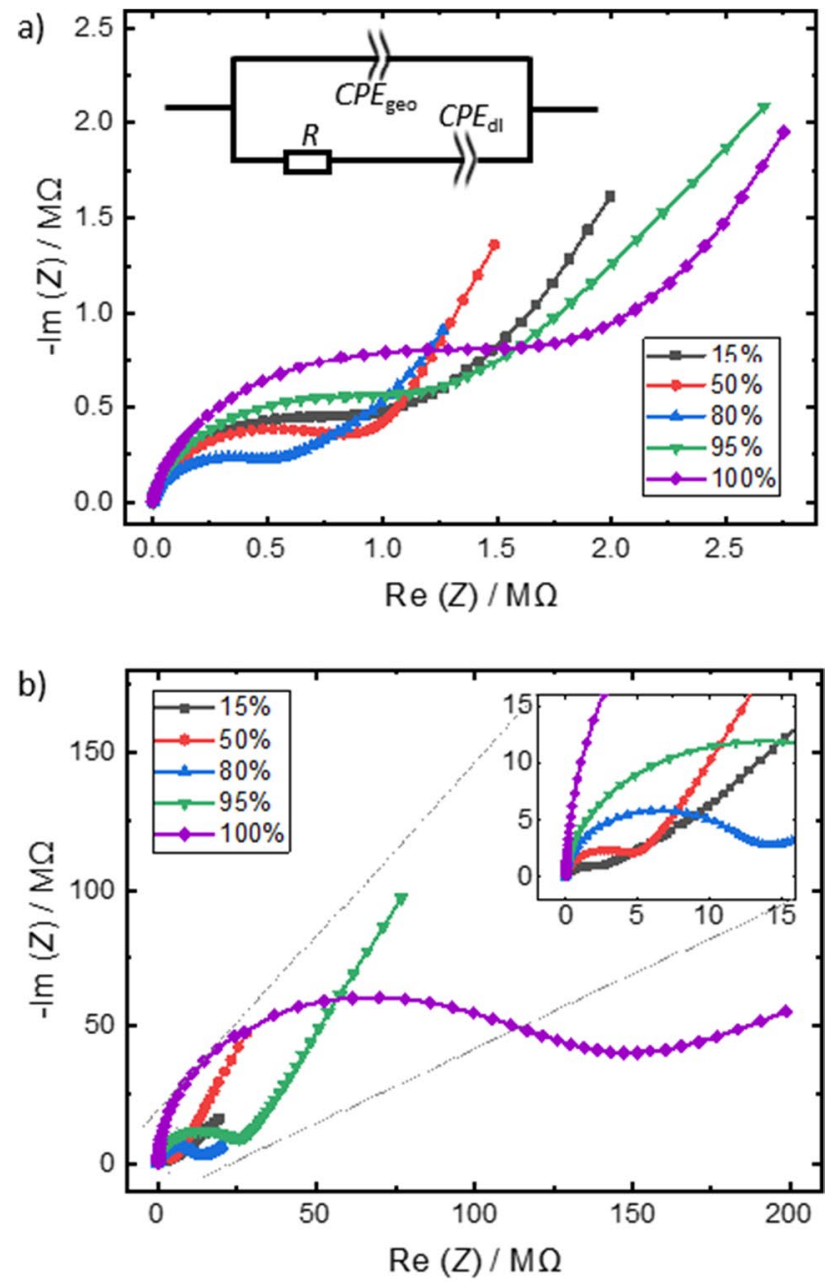

c)

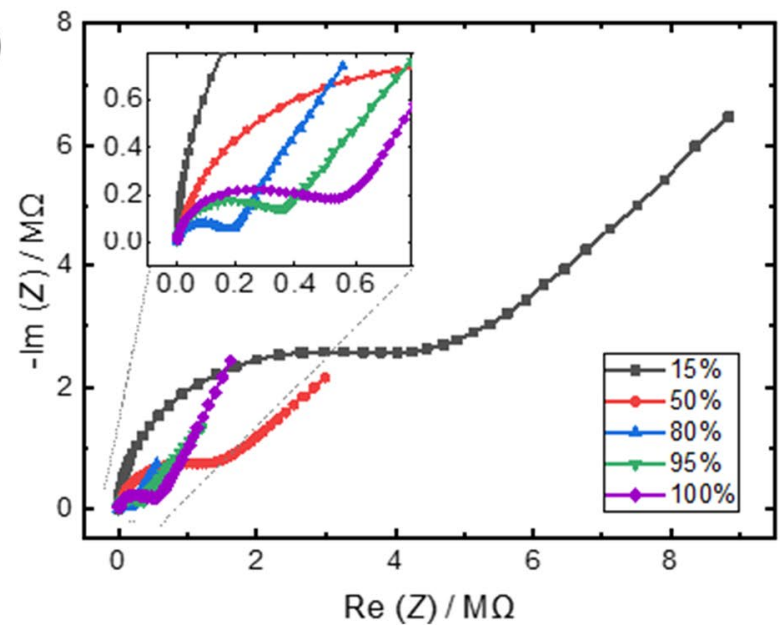

Fig. 5 Nyquist plots of the impedance of the HKUST-1 SURMOF film a upon IL loading without rinsing the surface, i.e., IL@SURMOF with excess IL on the surface, and $\mathbf{b}$ upon IL loading after shortly rinsing the surface, i.e., IL@SURMOF without excess IL on the surface. c Nyquist plots of the impedance of the IL@MOF-pellets with different IL loadings. The degree of IL filling is given in the legends. Zoom-ins of the Nyquist plots are shown in the insets. The equivalent circuit for the analysis is pictured in panel (a). The frequency range is $0.5 \mathrm{~Hz}$ to $5 \mathrm{MHz}$ for the SURMOF (a and b) and $100 \mathrm{~Hz}$ to $5 \mathrm{MHz}$ for the MOF pellet (c)

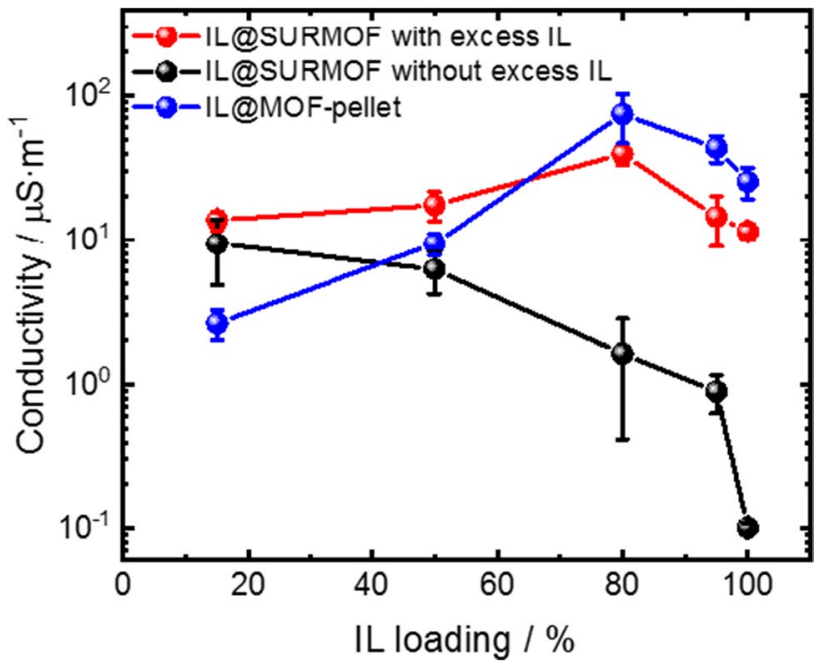

Fig. 6 Conductivity of [BMIM][TFSI] in HKUST-1 SURMOF film without excess IL and with excess IL on the surface and IL@MOFpellet with different IL fillings

Supplementary Information The online version contains supplementary material available at https://doi.org/10.1007/s11581-021-04249-w.

Acknowledgements We thank Beatriz Arevalo Galvan (IFG, KIT) for support with the contact angle measurements.

Funding Open Access funding enabled and organized by Projekt DEAL. The authors acknowledge the financial support of the German Science Foundation (HE 7036/5, and SPP 1928 "COORNETs") and the Chinese Scholarship Council (CSC).

Open Access This article is licensed under a Creative Commons Attribution 4.0 International License, which permits use, sharing, adaptation, distribution and reproduction in any medium or format, as long as you give appropriate credit to the original author(s) and the source, provide a link to the Creative Commons licence, and indicate if changes were made. The images or other third party material in this article are included in the article's Creative Commons licence, unless indicated otherwise in a credit line to the material. If material is not included in the article's Creative Commons licence and your intended use is not permitted by statutory regulation or exceeds the permitted use, you will need to obtain permission directly from the copyright holder. To view a copy of this licence, visit http://creativecommons.org/licenses/by/4.0/.

\section{References}

1. Welton T (1999) Room-temperature ionic liquids. Solvents for synthesis and catalysis. Chem Rev 99(8):2071-83. https://doi.org/ $10.1021 / \mathrm{cr} 980032 \mathrm{t}$

2. Wasserscheid P, Keim W (2000) Ionic liquids - New "solutions" for transition metal catalysis. Angew Chem, Int Ed 39(21):3772-3789. https://doi.org/10.1002/15213773(20001 103)39:21\%3c3772::Aid-anie 3772\%3e3.0. Co;2-5

3. MacFarlane DR, Kar M, Pringle JM (2017) Fundamentals of ionic liquids: from chemistry to applications. Wiley 
4. Salanne M (2017) Ionic liquids for supercapacitor applications. Top Curr Chem 375(3):25. https://doi.org/10.1007/ s41061-017-0150-7

5. Osada I, de Vries H, Scrosati B, Passerini S (2016) Ionic-liquidbased polymer electrolytes for battery applications. Angew ChemInt Edit 55(2):500-513. https://doi.org/10.1002/anie.201504971

6. MacFarlane DR, Tachikawa N, Forsyth M, Pringle JM, Howlett PC, Elliott GD et al (2014) Energy applications of ionic liquids. Energy Environ Sci 7(1):232-250. https://doi.org/10.1039/c3ee4 2099j

7. Furukawa H, Cordova KE, O'Keeffe M, Yaghi OM (2013) The chemistry and applications of metal-organic frameworks. Science 341(6149):1230444. https://doi.org/10.1126/science.1230444

8. Kaskel S (2016) The chemistry of metal-organic frameworks: synthesis, characterization, and applications. Wiley

9. Vaid TP, Kelley SP, Rogers RD (2017) Structure-directing effects of ionic liquids in the ionothermal synthesis of metal-organic frameworks. IUCrJ 4:380-392. https://doi.org/10.1107/s2052 252517008326

10. Kinik FP, Uzun A, Keskin S (2017) Ionic liquid/metal-organic framework composites: from synthesis to applications. Chemsuschem 10(14):2842-2863. https://doi.org/10.1002/cssc.201700716

11. Fujie K, Kitagawa H (2016) Ionic liquid transported into metalorganic frameworks. Coord Chem Rev 307:382-390. https://doi. org/10.1016/j.ccr.2015.09.003

12. Fujie K, Yamada T, Ikeda R, Kitagawa H (2014) Introduction of an ionic liquid into the micropores of a metal-organic framework and its anomalous phase behavior. Angew Chem-Int Edit 53(42):11302-11305. https://doi.org/10.1002/anie.201406011

13. Fujie K, Otsubo K, Ikeda R, Yamada T, Kitagawa H (2015) Low temperature ionic conductor: ionic liquid incorporated within a metal-organic framework. Chem Sci 6(7):4306-4310. https://doi. org $/ 10.1039 / \mathrm{c} 5 \mathrm{sc} 01398 \mathrm{~d}$

14. Tuffnell JM, Morzy JK, Kelly ND, Tan R, Song QL, Ducati C et al (2020) Comparison of the ionic conductivity properties of microporous and mesoporous MOFs infiltrated with a Na-ion containing IL mixture. Dalton Trans 49(44):15914-15924. https://doi. org/10.1039/d0dt02576c

15. Wang ZQ, Tan R, Wang HB, Yang LY, Hu JT, Chen HB et al (2018) A metal-organic-framework-based electrolyte with nanowetted interfaces for high-energy-density solid-state lithium battery. Adv Mater 30(2):7. https://doi.org/10.1002/adma.201704436
16. Yoshida Y, Fujie K, Lim D-W, Ikeda R, Kitagawa H (2019) Superionic conduction over a wide temperature range in a metalorganic framework impregnated with ionic liquids. Angew Chem, Int Ed 58(32):10909-10913. https://doi.org/10.1002/anie.20190 3980

17. Chen N, Li YJ, Dai YJ, Qu WJ, Xing Y, Ye YS et al (2019) A $\mathrm{Li}^{+}$conductive metal organic framework electrolyte boosts the high-temperature performance of dendrite-free lithium batteries. J Mater Chem A 7(16):9530-9536. https://doi.org/10.1039/c8ta1 $2539 \mathrm{~b}$

18. Chui SSY, Lo SMF, Charmant JPH, Orpen AG, Williams ID (1999) A chemically functionalizable nanoporous material $\mathrm{Cu}$ 3(TMA)(2)(H2O)(3) (n). Science 283(5405):1148-1150. https:// doi.org/10.1126/science.283.5405.1148

19. Al-Janabi N, Hill P, Torrente-Murciano L, Garforth A, Gorgojo $\mathrm{P}$, Siperstein F et al (2015) Mapping the Cu-BTC metal-organic framework (HKUST-1) stability envelope in the presence of water vapour for CO2 adsorption from flue gases. Chem Eng J 281:669677. https://doi.org/10.1016/j.cej.2015.07.020

20. Tagliabue M, Rizzo C, Millini R, Dietzel PDC, Blom R, Zanardi S (2011) Methane storage on CPO-27-Ni pellets. J Porous Mat 18(3):289-296. https://doi.org/10.1007/s10934-010-9378-0

21. Heinke L, Woll C (2019) Surface-mounted metal-organic frameworks: crystalline and porous molecular assemblies for fundamental insights and advanced applications. Adv Mater 31(26): 12. https://doi.org/10.1002/adma.201806324

22. Kanj AB, Verma R, Liu MD, Helfferich J, Wenzel W, Heinke L (2019) Bunching and immobilization of ionic liquids in nanoporous metal-organic framework. Nano Lett 19(3):2114-2120. https://doi.org/10.1021/acs.nanolett.8b04694

23. Liu JX, Wachter T, Irmler A, Weidler PG, Gliemann H, Pauly F et al (2015) Electric transport properties of surface-anchored metal-organic frameworks and the effect of ferrocene loading. ACS Appl Mater Interfaces 7(18):9824-9830. https://doi.org/10. 1021/acsami.5b01792

24. Falcaro P, Ricco R, Doherty CM, Liang K, Hill AJ, Styles MJ (2014) MOF positioning technology and device fabrication. Chem Soc Rev 43(16):5513-5560. https://doi.org/10.1039/c4cs00089g

Publisher's note Springer Nature remains neutral with regard to jurisdictional claims in published maps and institutional affiliations. 